\title{
INNER PRODUCT SPACES AND THE TRI-SPHERICAL INTERSECTION PROPERTY ${ }^{1}$
}

\author{
W. W. COMFORT AND HUGH GORDON ${ }^{2}$
}

1. Introduction and definitions. This note adds another criterion to the lengthy list of those properties which characterize the real inner product spaces whose dimension exceeds two. For a concise guide to the history of the development of this subject, the reader is referred to Day [2, VII, §3].

1.1. Definition. A subset $F$ of a linear space $E$ is said to be a flat if, for some $x \in E$, the set $F-x$ is a linear subspace of $E$.

1.2. Definition. Let $x_{1}, x_{2}$ and $x_{3}$ be points in a normed linear space $E$. Then $F\left(x_{1}, x_{2}, x_{3}\right)$ denotes the smallest flat containing each of the points $x_{i}$.

1.3. Definition. If $E$ is a normed linear space and $x \in E$ and $\rho>0$, then by $S_{\rho}(x)$ we mean the set $\{y \in E \mid\|x-y\| \leqq \rho\}$.

1.4. Definition. A normed linear space $E$ is said to have the trispherical intersection property if, whenever $x_{1}, x_{2}$ and $x_{3}$ are points in $E$ and $\rho_{1}, \rho_{2}$ and $\rho_{3}$ are positive numbers with $\bigcap_{i=1}^{3} S_{\rho_{i}}\left(x_{i}\right) \neq \varnothing$, it follows that $\bigcap_{i=1}^{3} S_{\rho_{i}}\left(x_{i}\right) \cap F\left(x_{1}, x_{2}, x_{3}\right) \neq \varnothing$.

1.5. REMARK. Every two-dimensional real normed linear space has the tri-spherical intersection property. Every real normed linear space has the bi-spherical intersection property, defined by analogy with 1.4 .

2. Theorems from the literature. We list here three theorems upon which the proof of our result depends.

2.1. Definition. (Birkhoff. See [1, p. 169].) If $x$ and $y$ are elements of a real normed linear space $E$, then we say $y$ is orthogonal to $x$, and write $y \perp x$, if $\|y-\alpha x\| \geqq\|y\|$ for each real number $\alpha$. If $J$ is a subset of $E$, then we write $J \perp x$ if $y \perp x$ for each $y \in J$.

2.2. Theorem. (James. See [5, Theorem 4].) A real normed linear space whose dimension exceeds 2 is an inner product space provided that for each hyperplane $H$ of $E$ containing $\phi$ there is a point $x$ of $E$ for which $x \neq \phi$ and $H \perp x$.

2.3. Theorem. (Fréchet. See $[3$, p. 717].) In order that a real

Presented to the Society, January 25, 1961; received by the editors May 20, 1960.

${ }^{1}$ Research supported in part by Office of Ordnance Research, U. S. Army, under contract DA-19-020-ORD-3778.

2 This paper arose out of a conversation with A. M. Gleason. 
normed linear space be an inner product space, it is sufficient that each of its three-dimensional subspaces be an inner product space.

2.4. Theorem. (Helly. See [4].) Let $D$ be an n-dimensional real normed linear space, and let $\mathcal{F}$ be a collection of compact, convex subsets of $D$. If every $n+1$ elements of $\mathcal{F}$ have a point in common, then some point of $D$ lies in every element of $\mathcal{F}$.

\section{Characterization of inner product space.}

3.1. TheOREM. Let $E$ be a real normed linear space of dimension $\geqq 3$. Then the following assertions are equivalent:

(a) $E$ is an inner product space,

(b) E has the tri-spherical intersection property.

Proof. $(\mathrm{a}) \Rightarrow(\mathrm{b})$. Let $x_{1}, x_{2}$ and $x_{3}$ be points in $E$, and let $z \in \bigcap_{i=1}^{3} S_{\rho_{i}}\left(x_{i}\right)$ for certain positive numbers $\rho_{i}$. The set $F\left(x_{1}, x_{2}, x_{3}\right)$, being complete in $E$, is closed in the completion $\bar{E}$ of $E$. Clearly we may suppose that $\phi \in F\left(x_{1}, x_{2}, x_{3}\right)$. Let $P$ denote the orthogonal projection of $\bar{E}$ onto $F\left(x_{1}, x_{2}, x_{3}\right)$. Then either each $x_{i}=\phi$ or $\|P\|=1$, and in any event we have $\left\|x_{i}-P(z)\right\|=\left\|P\left(x_{i}-z\right)\right\| \leqq\left\|x_{i}-z\right\| \leqq \rho_{i}$ for each $i$. Thus $P(z) \in \bigcap_{i=1}^{3} S_{p_{i}}\left(x_{i}\right)$.

(b) $\Rightarrow$ (a). Let $D$ be a three-dimensional linear subspace of $E$, and let $H$ be a two-dimensional linear subspace of $D$. In view of 2.2 and 2.3 , we need only show that $H \perp x$ for some $x \in D$ with $x \neq \phi$. Choose any $z \in D$ which is not in $H$. Define $\mathcal{F}=\left\{S_{\rho}(y) \mid \rho>0, y \in H\right.$ and $\|y-z\| \leqq \rho\}$. Since $D$ inherits the tri-spherical intersection property from $E$, every three elements of $\mathcal{F}$ have a point in common which belongs to $H$. Hence by 2.4 there is a point $w \in H$ with the property that if $y \in H$ and $\|y-z\| \leqq \rho$, then $\|y-w\| \leqq \rho$, i.e., with the property that if $y \in H$, then $\|y-w\| \leqq\|y-z\|$. Now define $x=z-w$. Since $z \notin H$ and $w \in H$, we have $x \neq \phi$. Then for each nonzero scalar $\alpha$ and each $y \in H$ we have

$$
\|y / \alpha-x\|=\|(y / \alpha+w)-z\| \geqq\|(y / \alpha+w)-w\|=\|y / \alpha\| .
$$

Hence $\|y-\alpha x\| \geqq\|y\|$ and $H \perp x$.

3.2. Remark. From 2.4 with $n=2$ it follows that a real normed linear space $E$ has the tri-spherical intersection property if and only if the following statement is true: If $x_{1}, x_{2}$ and $x_{3}$ are points in $E$ and $\rho_{1}, \rho_{2}$ and $\rho_{3}$ are positive numbers with $\bigcap_{i=1}^{3} S_{\rho_{i}}\left(x_{i}\right) \neq \varnothing$, then $\bigcap_{i-1}^{3} S_{\rho_{i}}\left(x_{i}\right) \cap \operatorname{conv}\left(x_{1}, x_{2}, x_{3}\right) \neq \varnothing$. (By $\operatorname{conv}\left(x_{1}, x_{2}, x_{3}\right)$ we mean the smallest convex set containing each of the points $x_{i}$.)

3.3. REMARK. If the tri-spherical intersection property had been defined in 1.4 using open spheres of the form 


$$
S_{\rho}(x)=\{y \in E \mid\|x-y\|<\rho\},
$$

then the statement of 3.1 remains valid. A minor modification of the proof of the implication $(b) \Rightarrow(a)$ is required.

4. Characterization of ellipsoids. Let $E$ be a Euclidean space. Let $S$ be a compact convex body in $E$ symmetric about one of its points. We call this point the centre of $S$. To avoid complications, we shall define an ellipsoid in $E$ to be a convex body which determines a norm given by an inner product. We call a subset of $E$ homothetic to $S$ if it is of the form $\alpha S+x$ with $\alpha>0$ and $x \in E$. Our result now takes the following form: $S$ is not an ellipsoid if and only if there are sets $S_{1}, S_{2}$ and $S_{3}$, with centres $x_{1}, x_{2}$ and $x_{3}$, homothetic to $S$, such that $S_{1} \cap S_{2} \cap S_{3} \neq \varnothing$ but $S_{1} \cap S_{2} \cap S_{3} \cap \operatorname{conv}\left(x_{1}, x_{2}, x_{3}\right)=\varnothing$.

\section{REFERENCES}

1. Garrett Birkhoff, Orthogonality in linear metric spaces, Duke Math. J. vol. 1 (1935) pp. 169-172.

2. Mahlon M. Day, Normed linear spaces, Berlin, Springer-Verlag, 1958.

3. Maurice Fréchet, Sur la definition axiomatique d'une classe d'espaces vectoriels distancies applicables vectoriellement sur l'espace de Hilbert, Ann. of Math. vol. 36 (1935) pp. 705-718.

4. Ed. Helly, Über Mengen konvexer Körper mit gemeinschlaftlichen Punkten, Jber. Deutsch. Math. Verein. vol. 32 (1923) pp. 175-176.

5. R. C. James, Inner products in normed linear spaces, Bull. Amer. Math. Soc. vol. 53 (1947) pp. 559-566.

HaRVARd UNIVERSITY 